\title{
The Evolving Facets of Bacterial Vaginosis: Implications for HIV Transmission
}

\author{
Lyle R. McKinnon, ${ }^{1,2}$ Sharon L. Achilles, ${ }^{3,4}$ Catriona S. Bradshaw, ${ }^{5,6}$ Adam Burgener, ${ }^{7-9}$ Tania Crucitti, ${ }^{10}$ \\ David N. Fredricks, ${ }^{11,12}$ Heather B. Jaspan, ${ }^{13,14}$ Rupert Kaul, ${ }^{15,16}$ Charu Kaushic, ${ }^{17,18}$ Nichole Klatt, ${ }^{19}$ \\ Douglas S. Kwon, ${ }^{20,21}$ Jeanne M. Marrazzo, ${ }^{22}$ Lindi Masson, ${ }^{23,24}$ R. Scott McClelland, ${ }^{12,25,26}$ Jacques Ravel, ${ }^{27}$ \\ Janneke H.H.M. van de Wijgert, ${ }^{28,29}$ Lenka A. Vodstrcil, ${ }^{5,6}$ and Gilda Tachedjian ${ }^{30-33}$
}

\begin{abstract}
Bacterial vaginosis (BV) is a common yet poorly understood vaginal condition that has become a major focus of HIV transmission and immunology research. Varied terminologies are used by clinicians and researchers to describe microbial communities that reside in the female reproductive tract (FRT), which is driven, in part, by microbial genetic and metabolic complexity, evolving diagnostic and molecular techniques, and multidisciplinary perspectives of clinicians, epidemiologists, microbiologists, and immunologists who all appreciate the scientific importance of understanding mechanisms that underlie BV. This Perspectives article aims to clarify the varied terms used to describe the cervicovaginal microbiota and its "nonoptimal" state, under the overarching term of $\mathrm{BV}$. The ultimate goal is to move toward language standardization in future literature that facilitates a better understanding of the impact of BV on FRT immunology and risk of sexually transmitted infections, including HIV.
\end{abstract}

Keywords: HIV, vaginal microbiota, bacterial vaginosis, HIV transmission, genital inflammation, female reproductive tract

\footnotetext{
${ }^{1}$ Department of Medical Microbiology and Infectious Diseases, University of Manitoba, Winnipeg, Canada.

${ }^{2}$ Centre for the AIDS Programme of Research in South Africa, Durban, South Africa.

${ }^{3}$ Department of Obstetrics, Gynecology, and Reproductive Sciences, University of Pittsburgh, Pittsburgh, Pennsylvania.

${ }^{4}$ Magee-Womens Research Institute, Pittsburgh, Pennsylvania.

${ }^{5}$ Central Clinical School, Monash University, Melbourne, Australia.

${ }^{6}$ Melbourne Sexual Health Centre, Alfred Hospital, Carlton, Australia.

${ }^{7}$ National HIV and Retrovirology Labs, Public Health Agency of Canada, Winnipeg, Canada.

${ }^{8}$ Departments of Obstetrics and Gynecology, and Medical Microbiology, University of Manitoba, Winnipeg, Canada.

${ }^{9}$ Department of Medicine Solna, Karolinska Institute, Stockholm, Sweden.

${ }^{10}$ Centre Pasteur du Cameroun, Yaoundé, Cameroon.

${ }^{11}$ Vaccine and Infectious Diseases, Fred Hutchinson Cancer Research Center, Seattle, Washington.

${ }^{12}$ Department of Medicine, University of Washington, Seattle, Washington.

${ }^{13}$ Seattle Children's Research Institute and University of Washington, Seattle, Washington.

${ }^{14}$ Department of Pathology, Division of Immunology, Institute of Infectious Disease and Molecular Medicine, University of Cape Town, Cape Town, South Africa.

Departments of ${ }^{15}$ Immunology and ${ }^{16}$ Medicine, University of Toronto, Toronto, Canada.

${ }^{17}$ McMaster Immunology Research Centre, Michael G. DeGroote Centre for Learning and Discovery, McMaster University, Hamilton, Canada.

${ }^{18}$ Department of Pathology and Molecular Medicine, McMaster University, Hamilton, Canada.

${ }^{19}$ Department of Pediatrics, University of Miami, Miami, Florida.

${ }^{20}$ Ragon Institute of MGH, MIT, and Harvard, Massachusetts General Hospital, Cambridge, Massachusetts.

${ }^{21}$ Harvard Medical School, Boston, Massachusetts.

${ }^{22}$ Division of Infectious Disease, University of Alabama at Birmingham, Birmingham, Alabama.

${ }^{23}$ Division of Medical Virology, Institute of Infectious Disease and Molecular Medicine, University of Cape Town, Cape Town, South Africa.

${ }^{24}$ Centre for AIDS/HIV Program of Research in South Africa (CAPRISA) Centre of Excellence, University of Cape Town, Cape Town, South Africa.

Departments of ${ }^{25}$ Epidemiology and ${ }^{26}$ Global Health, University of Washington, Seattle, Washington.

${ }^{27}$ Institute for Genome Sciences and Department of Microbiology and Immunology, University of Maryland School of Medicine, Baltimore, Maryland.
} 


\section{Introduction}

B ACTERIA ARE NOW RECOGNIZED to play important immunological roles at all mucosal surfaces, and the female reproductive tract (FRT) is no exception. ${ }^{1}$ The entirety of "optimal" microbial communities associated with a mucosal site (i.e., the microbiota) is an important contributor to the effectiveness of the host mucosal barrier against infection. ${ }^{2}$ This is in contrast to "nonoptimal" microbial communities that are associated with the disruption of important physiological roles of bacteria at the mucosa. ${ }^{1}$ An example of nonoptimal microbiota is bacterial vaginosis (BV), a common vaginal condition in women of reproductive age associated with adverse urogenital and reproductive health outcomes, including an increased risk of HIV acquisition. ${ }^{3-7}$ BV affects $29 \%$ of women in the United States and 52\% of women in sub-Saharan Africa, where HIV is also highly prevalent. ${ }^{8}$

BV is commonly diagnosed by clinicians using Amsel's criteria, 9 defined here as "Amsel-BV," a "vaginal discharge syndrome" wherein at least three out of four diagnostic criteria need to be met (Box 1). While women with BV can present with a vaginal discharge, $\mathrm{BV}$ is not typically associated with redness, swelling, or pain seen with "overt" inflammation, ${ }^{10}$ which is why it is referred to as "vaginosis" rather than "vaginitis." However, BV is associated with "subclinical" genital inflammation, as determined by an increase in proinflammatory cytokines and chemokines ${ }^{10-16}$ associated with increased HIV risk. ${ }^{17-19}$ A second common method used to diagnose BV is by Nugent score, defined here as "Nugent-BV" (Box 1). ${ }^{20}$ The Nugent score captures bacterial morphotypes on a Gram stain, differentiating Lactobacillus-dominated bacterial communities from the presence of small Gram-variable rods (Gardnerella vaginalis morphotypes) and curved Gram-variable rods (Mobiluncus spp. morphotypes), ${ }^{5}$ which is an oversimplification of the actual ecology of BV. ${ }^{21,22}$

Nugent scoring has been used widely, particularly in epidemiology research, to define BV in large cohort studies, correlating $\mathrm{BV}$ to a wide range of adverse health outcomes. ${ }^{18,19,23}$ A proportion of women with Nugent-BV are clinically asymptomatic ("asymptomatic BV"). Nugent-BV can be sustained or transient, the latter representing a temporary shift in the vaginal microbiota mediated by intrinsic (menses) or extrinsic (sex) factors, ${ }^{24}$ which may or may not be associated with increased HIV risk. Some women with Amsel-BV may also not present with symptoms. While this may be uncommon for women presenting to a clinic, population-based Amsel screening will identify asymptomatic Amsel-BV-positive women. The presence of signs and symptoms of BV vary widely based on the perception of women and clinicians, complicating its diagnostic usefulness. Thus, BV that is diagnosed by either Amsel or Nugent methods can be further delineated as either "asymptomatic" or "symptomatic."
While clinical manifestations of $\mathrm{BV}$ are important for patient care, it is now clear with advances in DNA sequencing technology that a broader range of nonoptimal cervicovaginal microbiota have relevance for adverse sexual and reproductive health outcomes. Cervicovaginal microbiota are genetically and ecologically complex, diverse, and dynamic. ${ }^{24}$ This combined with its health implications has made it a "hot topic" for molecular microbiologists. Several immunological and clinical associations of various cervicovaginal bacterial communities have now been characterized using molecular methods; these "nonoptimal" microbiota broadly overlap with BV defined by other methods, but are distinct, and we have termed these "Molecular-BV" (Box 1). As subtle differences between methods come to light, "Molecular-BV" should be further subdivided into terms that incorporate the specific molecular method (Box 1). One common method for microbiota characterization in recent literature is deep sequencing of the 16S rRNA gene. This method has been termed as a "broad-range PCR" method that measures the relative abundance of bacteria taxa without preconceived knowledge of the bacteria that are present. ${ }^{25-28}$ We propose that "nonoptimal" microbial communities defined by this technique be designated as "Seq-BV," which would also incorporate whole-genome shotgun sequencing approaches. A second method is taxon-specific quantitative PCR (qPCR) that quantifies the absolute abundance of predetermined taxa, ${ }^{26,29}$ while not including others. We propose that "nonoptimal" taxa are designated as "qPCR-BV." Metaproteomic analysis of cervicovaginal samples has also been employed to study the cervicovaginal environment, including bacterial composition, which has led to "optimal" and "nonoptimal" bacterial community classifications, where the latter could be designated as Prot-BV. ${ }^{30-32}$

The concept of "Molecular-BV," as defined currently in research settings, is intended to be an "overarching" term to describe nonoptimal cervicovaginal microbiota characterized by molecular methods. This is not to suggest that it is not clinically relevant. An FDA-approved molecular diagnostic test for BV is being used in the United States. ${ }^{33,34}$ In addition, Molecular-BV has been associated with genital inflammation and/or adverse sexual and reproductive health outcomes, such as increased HIV risk, ${ }^{17,29}$ and therefore is prognostic for clinical outcomes. Understanding this distinction may enable better comparisons to studies where BV has been determined using Amsel's criteria or Nugent score. ${ }^{18,19}$

Molecular-BV bacterial communities are depleted of Lactobacillus spp., with a high relative abundance or load of facultative and/or obligate anaerobes (see Box 1 and Table 1 for microbial communities typical of Molecular-BV). ${ }^{12,17,25-27}$ These communities are usually "highly diverse" (i.e., high species richness or polymicrobial) and show "evenness" (i.e.,

\footnotetext{
${ }^{28}$ Julius Center for Health Sciences and Primary Care, University Medical Center Utrecht, Utrecht, the Netherlands.

${ }^{29}$ Institute of Infection and Global Health, University of Liverpool, Liverpool, United Kingdom.

${ }^{30}$ Disease Elimination Program, Life Sciences Discipline, Burnet Institute, Melbourne, Australia.

${ }^{31}$ Department of Microbiology, Monash University, Clayton, Australia.

${ }^{32}$ Department of Microbiology and Immunology at the Peter Doherty Institute for Infection and Immunity, The University of Melbourne, Melbourne, Australia.

${ }^{33}$ School of Science, College of Science, Engineering and Health, RMIT University, Melbourne, Australia.

(C) Lyle R. McKinnon et al. 2019; Published by Mary Ann Liebert, Inc. This Open Access article is distributed under the terms of the Creative Commons License (http://creativecommons.org/licenses/by/4.0), which permits unrestricted use, distribution, and reproduction in any medium, provided the original work is properly cited.
} 


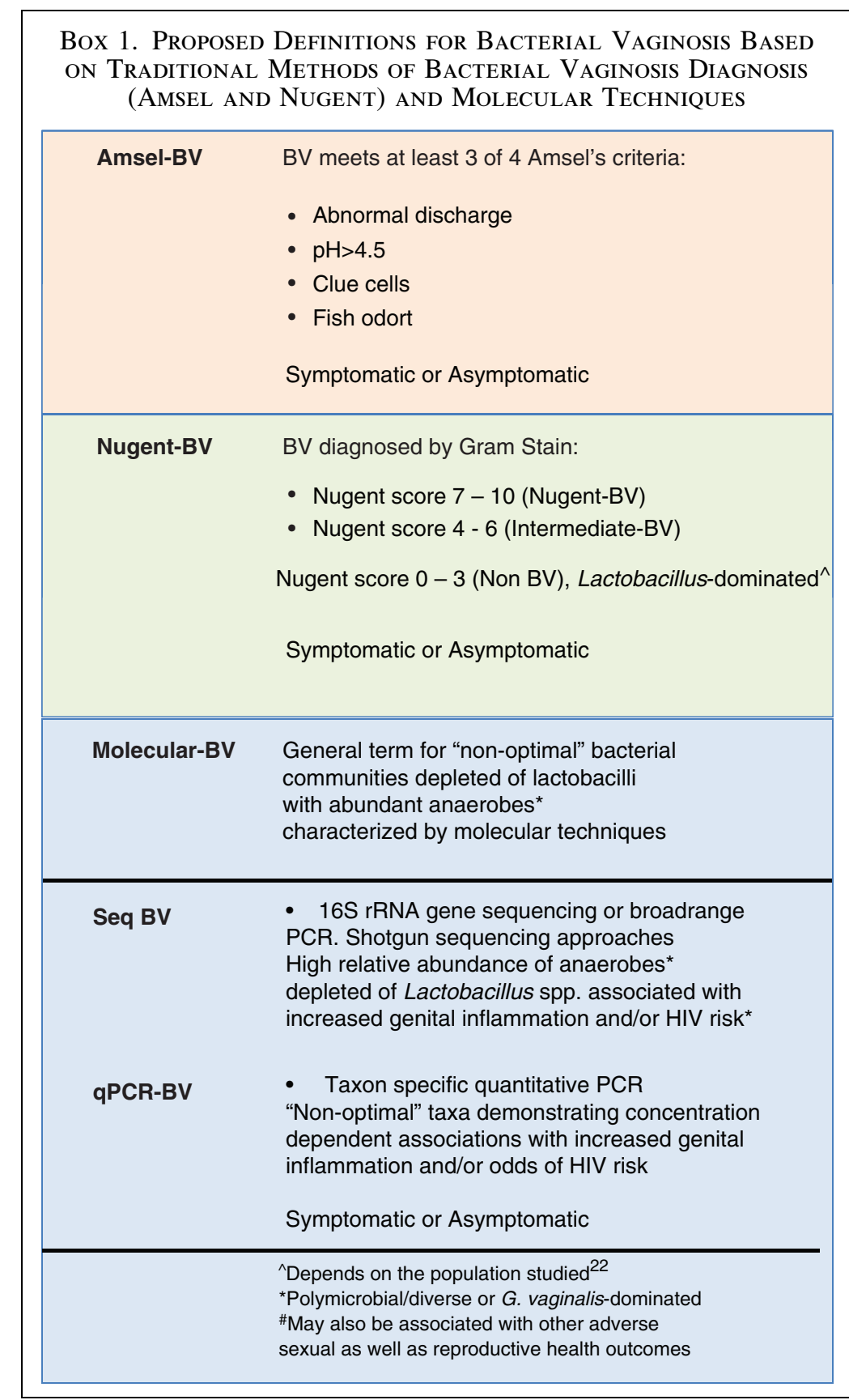

Color images are available online.

not dominated by particular species), although they can be dominated by one species. Examples of Seq-BV include microbiota commonly referred to as cervicotypes 3 (CT3) and 4 (CT4), which proportionally are depleted of Lactobacillus spp. and predominately contain $G$. vaginalis or an increase in a mixture of diverse anaerobes comprising Prevotella, Gardnerella, BVAB1, Sneathia, and Megasphera spp., respectively. ${ }^{11,17}$ Examples of qPCR-BV include additional taxa shown to have a concentration-dependent association with genital inflammation and/or increased risk of HIV acquisition, for example, Gemella asaccharolytica and Eggerthella species type I. ${ }^{29}$

Techniques used to define Molecular-BV have demonstrated that an even larger proportion of asymptomatic women may be at risk of subclinical cervicovaginal inflammation and increased risk of acquiring sexually transmitted infections (STIs), including HIV. ${ }^{17,29}$ However, these overlapping yet distinct approaches for defining BV have led to some confusion among researchers in the field. A patchwork of terms describing BV and cervicovaginal microbiota continues to evolve as studies employ increasingly complex molecular measurements to better capture aspects of the microbiota that go beyond clinical or microscopic criteria by using bacterial relative or absolute bacterial abundances. This Perspectives article attempts to capture the heterogeneous terminology generated from this multidisciplinary research effort geared at understanding the intricate relationships between " $\mathrm{BV}$," as defined by Amsel, Nugent and molecular methods, cervicovaginal inflammation, and the risk of HIV/STIs. 
Table 1. Classification of Cervicovaginal Bacterial Communities Determined BY 16S RRNA GENE SEQUENCING

\begin{tabular}{|c|c|c|}
\hline Abbreviation & Definition & $\begin{array}{l}\text { Molecular-BV } \\
\quad(\text { Seq- } B V)\end{array}$ \\
\hline CST-I & Lactobacillus crispatus-dominated & No \\
\hline CST-II & Lactobacillus gasseri-dominated & No \\
\hline CST-III & Lactobacillus iners-dominated & No \\
\hline CST-IVA & $\begin{array}{l}\text { Modest Lactobacillus spp., higher relative abundance of facultative } \\
\text { and/or obligate anaerobes, BVABI and Gardnerella vaginalis }\end{array}$ & Yes \\
\hline CST-IVB & $\begin{array}{l}\text { Modest Lactobacillus spp., higher relative abundance of facultative } \\
\text { and/or obligate anaerobes, G. vaginalis and Atopobium vaginae }\end{array}$ & Yes \\
\hline CST-IVC & $\begin{array}{l}\text { Lacking Lactobacillus spp. and more even in anaerobe composition } \\
\text { (i.e., no bacteria dominate) comprising Prevotella among others, } \\
\text { as well as Anaerococcus, Finegoldia, Corynebacterium, } \\
\text { Peptoniphilus, Megasphaera, and Gemella spp. }\end{array}$ & Yes \\
\hline CST-V & Lactobacillus jensenii-dominated & No \\
\hline CT1 & L. crispatus-dominated & No \\
\hline $\mathrm{CT} 2$ & L. iners-dominated & No \\
\hline CT3 & Depleted of Lactobacillus spp. and G. vaginalis-dominated & Yes \\
\hline CT4 & $\begin{array}{l}\text { Depleted Lactobacillus spp. and polymicrobial with a higher } \\
\text { relative abundance of facultative and/or obligate anaerobes } \\
\text { comprising Prevotella, Gardnerella, BVAB1, Sneathia, } \\
\text { and Megasphera spp. }\end{array}$ & Yes \\
\hline $\mathrm{C} 1$ & $\begin{array}{l}\text { Depleted of Lactobacillus spp. and polymicrobial with a higher } \\
\text { relative abundance of facultative and/or obligate anaerobes }\end{array}$ & Yes \\
\hline $\mathrm{C} 2$ & L. crispatus-dominated & No \\
\hline $\mathrm{C} 3$ & L. iners-dominated & No \\
\hline
\end{tabular}

C, compositional subtype ${ }^{12,52}$; CST, community state type ${ }^{24,25}$; CT, cervicotype ${ }^{11,17}$; Molecular-BV, bacterial vaginosis determined by characterizing vaginal or cervical samples using molecular methods; Seq-BV, BV as determined by 16S rRNA gene sequencing.

While here we focus on BV, it is important to note that there are additional forms of "nonoptimal" cervicovaginal microbiota associated with vulvovaginal candidiasis (VVC) caused by Candida spp., and desquamative vaginitis or aerobic vaginitis caused by pathobionts Proteobacteria, Streptococci, Staphylococci, or Enterococci spp. ${ }^{35,36}$ These microbes and STIs other than HIV are clinically relevant and are associated with genital inflammation that can increase HIV risk (Fig. 1) and are, therefore, important when considering sources of inflammation in the cervicovaginal mucosa, but do not feature in the definitions of $\mathrm{BV}$, which is the current focus of this Perspectives article. ${ }^{35}$ An update on this topic is planned in a report on the 2018 Keystone Symposia on the Role of the Genital Tract Microbiome in Sexual and Reproductive Health.

\section{Partial Overlap Between Amsel-BV, Nugent-BV, and Molecular-BV}

Current evidence supports that only a minority of BV is symptomatic. Molecular-BV/Seq-BV, which categorizes microbiota into bacterial community types (Table 1), tends to correlate with vaginal $\mathrm{pH}$ and not with other Amsel criteria, such as clue cells and whiff test. ${ }^{28,37}$ Similarly, although Seq-BV correlates with Nugent-BV (Fig. 2), the overlap is incomplete. $^{17,22,25}$ The majority of women who have an intermediate Nugent score (defined in Box 1) also have Seq-BV (e.g., CT3, CT4, and CST-IV), ${ }^{11,17,22}$ indicating an association with adverse health outcomes. ${ }^{17}$ We propose that data from primarily clinical (Amsel), microscopic (Nugent), and molecular evaluation of BV fit into an "iceberg" concept of a clinical/subclinical condition (Fig. 2). Amsel-BV is at the top of the iceberg, usually capturing clinically apparent nonoptimal vaginal microbiota, while both Nugent-BV and Molecular-BV include additional microbial states that can be subclinical (e.g., asymptomatic) but still clinically relevant for infection and/or health risk. It is worth noting that some Amsel-positive diagnoses may not be Nugent-positive due to either subjectivity of the Amsel criteria (e.g., vaginal discharge, odor) or perhaps differences in the ability of these tests to detect BV associated with biofilm versus planktonic $\mathrm{BV}$, although these cases are uncommon.

\section{Nonoptimal Cervicovaginal Microbiota, Genital Inflammation, and HIV Acquisition Risk}

Meta-analyses clearly demonstrate that women with Nugent-BV and/or Amsel-BV have an increased risk of acquiring HIV. ${ }^{18,19}$ Meta-analyses by Atashili et al. of 23 studies, including 30,739 women, reported a relative risk of 1.61 [95\% confidence interval $(\mathrm{CI})=1.21-2.13$ ] for HIV acquisition in women with Nugent-BV. ${ }^{18}$ Subsequently, an individual patient meta-analysis by Low et al. reported that Nugent-BV, measured at the seronegative visit before HIV diagnosis, was associated with an adjusted hazard ratio of 1.53 (95\% CI = 1.24-1.89) for HIV acquisition risk. ${ }^{19}$ This study also demonstrated an elevated susceptibility to HIV (adjusted hazard ratio $=1.41 ; 95 \% \mathrm{CI}=1.12-1.79$ ) in women with intermediate Nugent scores, ${ }^{19}$ suggesting that any 


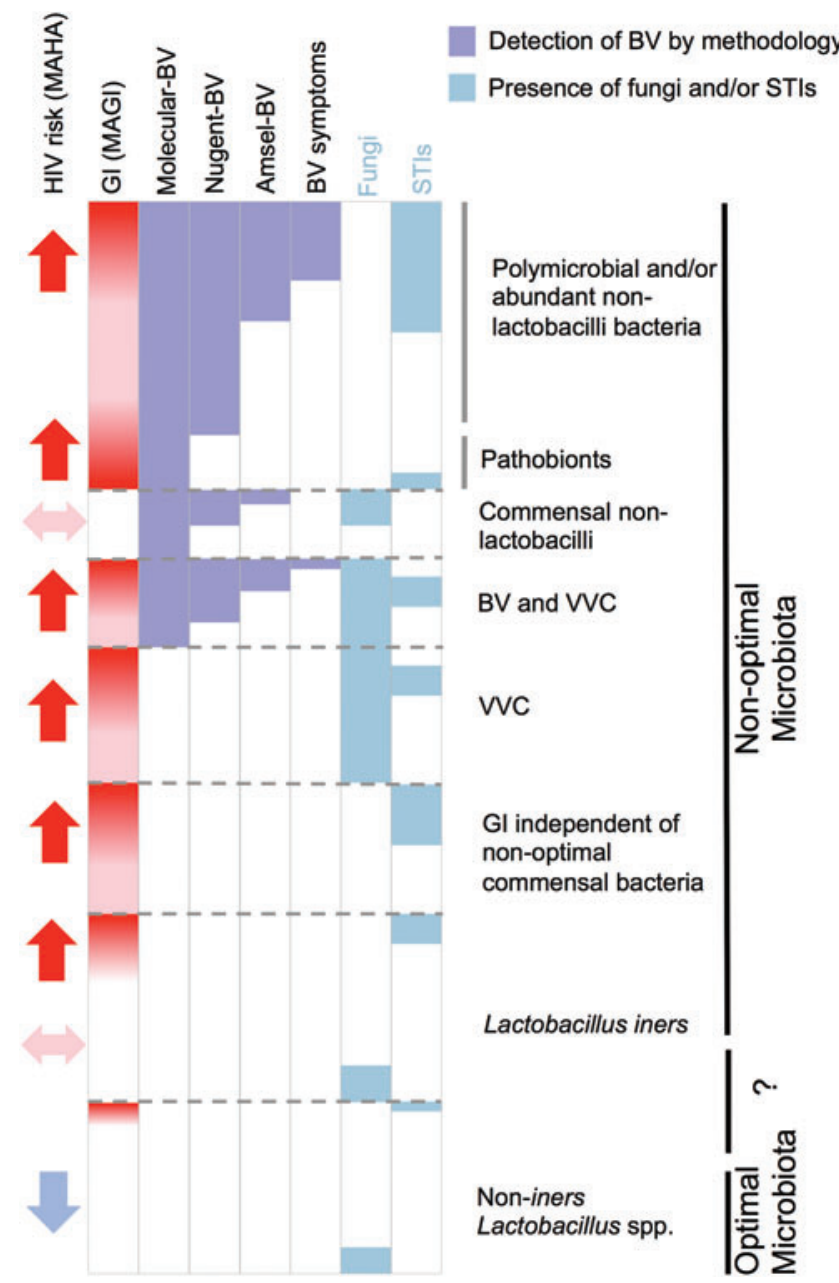

FIG. 1. Microbial causes of genital inflammation and/or altered HIV susceptibility. Each microbial class can cause inflammation independently or in combination with other microorganisms that may also be present in the same women. Strategies to mitigate as many of these causes as possible may be key to achieving the optimal FRT mucosa associated with positive health outcomes, including protection against HIV infection. Optimal - cervicovaginal microbiota associated with no vaginal symptoms, lack of genital inflammation, and decreased HIV risk; nonoptimal - cervicovaginal microbiota associated with vaginal symptoms and/or genital inflammation and/or increased HIV risk. BV, bacterial vaginosis; FRT, female reproductive tract; GI, genital inflammation; MAGI, microbiota associated with genital inflammation; MAHA, microbiota associated with HIV acquisition; pathobionts, a symbiotic organism under normal circumstances that can become pathogenic, for example, Proteobacteria, Streptococci, Staphylococci, or Enterococci spp.; STIs, sexually transmitted infections; VVC, vulvovaginal candidiasis; Lactobacillus spp. (e.g., Lactobacillus crispatus) or strains that may not be optimal (e.g. Lactobacillus iners). Color images are available online.

Nugent score $>3$ may be a risk factor for HIV. On the basis of more recent molecular studies, a large proportion of these women would be expected to have Molecular-BV. ${ }^{11,17,22}$

Nugent-BV has repeatedly been associated with genital inflammation; in particular, proinflammatory cytokines are typically upregulated, whereas chemokines show no association, are upregulated (i.e., IL-8), or downregulated. ${ }^{10,14-16}$ This cytokine-chemokine distinction is likely due to the ob-

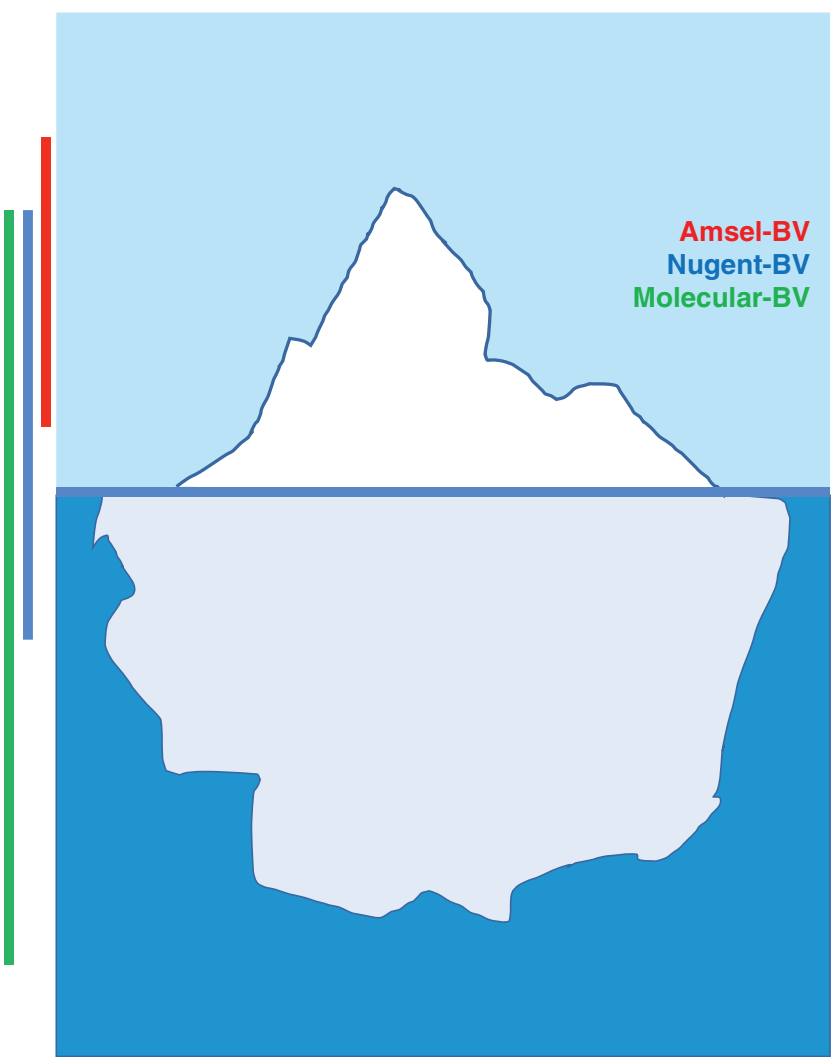

FIG. 2. The "clinical iceberg" concept of adverse health outcomes, applied to BV. With better molecular methods, we now appreciate that clinically evident $\mathrm{BV}$, as diagnosed by a technique such as Amsel's criteria (Amsel-BV), does not capture a high proportion of women diagnosed with BV by Nugent (Nugent-BV) or with molecular methods (MolecularBV) that contributes to adverse sexual and reproductive health outcomes, including increased HIV risk. Not all Amsel-BVpositive samples are Nugent-BV- or Molecular-BV-positive. Red vertical line denotes Amsel-BV, blue vertical line denotes Nugent-BV, and green vertical line denotes Molecular-BV. Color images are available online.

servation that BV is microbiologically multifaceted, and specific combinations of bacterial species may result in different host responses. ${ }^{28}$ The host response to the same bacterial communities could also vary between individuals, even though no studies have evaluated this specific question. In addition, some differences could be accounted for by methodological differences in sampling and measuring immune mediators in the genital tract. ${ }^{10}$

Molecular-BV has often been associated with both genital inflammation $^{11-13}$ and an increased risk of HIV acquisition. ${ }^{17,29}$ A prospective study in South Africa reported that young women colonized with a highly diverse community (CT4) had a 4.4-fold (95\% CI $=1.17-16.61)$ increased risk of acquiring HIV compared with women with Lactobacillus crispatus-dominant microbiota. ${ }^{17}$ Presence of G. vaginalisdominated (CT3) cervicotype demonstrated a trend toward elevated HIV risk, although it did not reach statistical significance after adjusting for the presence of chlamydia. ${ }^{17}$ The Lactobacillus iners-dominated (CT2) cervicotype was not significantly associated with increased HIV risk. ${ }^{17}$ In this cohort, women with CT4 also had the greatest genital inflammation 
measured by levels of proinflammatory cytokines and chemokines, compared with women with $L$. crispatus-dominated microbiota, and followed by $G$. vaginalis-dominated and L. inersdominated microbiota. ${ }^{11}$ Another nested case-control study in African women showed that vaginal bacterial diversity and several BV-associated bacterial species, including Parvimonas species types 1 and 2, G. asaccharolytica, Mycoplasma hominis, Leptotrichia/Sneathia, Eggerthella species type 1, and Megasphaera species, were significantly associated with higher risk of HIV acquisition. ${ }^{29}$

Several studies have demonstrated that elevated genital inflammation is associated with an increase in activated HIV target cells in the cervix, ${ }^{11,17,38}$ consistent with elevated HIV risk. ${ }^{17}$ However, not all studies have found an association between cervicovaginal bacterial communities and the frequency of $\mathrm{CD}^{+}$and $\mathrm{CCR}^{+}$activated or proliferating HIV target cells in the cervix, ${ }^{12,39}$ suggesting differences between geographic or ethnic populations. Alternatively, there could be other mechanisms by which nonoptimal cervicovaginal microbiota increase HIV risk, such as disruption of epithelial barrier integrity. ${ }^{30-32,38}$

\section{Effect Sizes for HIV Risk Determined by Nugent-BV/Amsel-BV Versus Molecular-BV}

The effect sizes for Nugent-BV/Amsel-BV on HIV risk are typically smaller, that is, $60 \%$ increased risk ${ }^{18,19}$ compared with the effect size from Seq-BV on HIV risk (i.e., >4fold). ${ }^{17,28}$ However, the latter was only from two studies, with modest number of women colonized with L. crispatusdominated cervicovaginal microbiota, and needs to be confirmed. ${ }^{17}$ The large sample size (>30,000 individuals) evaluated in the BV meta-analysis could contribute to and explain the smaller effect sizes. The meta-analysis of Nugent$\mathrm{BV}$ is also adjusted for potential confounders such as VVC and sexual behaviors, although these are incompletely controlled for in studies relying on Molecular-BV. ${ }^{17,29}$

While Nugent and Amsel are useful tools for epidemiological and clinical studies, they could be thought of as less sensitive, that is, underestimating the types of microbiota that put a woman at risk for HIV, compared with molecular evaluation of cervicovaginal microbiota, at least in research settings (Fig. 2). Nugent and molecular techniques can also detect brief episodes of "nonoptimal" microbiota (e.g., during menses) ${ }^{24}$ that may not cause significant genital inflammation and/or increase HIV risk, and it is likely that more broadly these communities may be dynamic, dependent upon a number of host and environmental factors (genital hygiene practices, sexual behavior, comorbidities, etc.). Therefore, the duration and frequency of "nonoptimal" vaginal microbiota are likely a critical factor requiring the incorporation of frequent sampling in longitudinal studies to better define the HIV risk associated with Nugent-BV and Molecular-BV.

\section{Not All Lactobacillus spp. Are Associated with Reduced Genital Inflammation and Protection Against HIV Acquisition}

Lactobacillus spp.-dominated cervicovaginal microbiota, and particularly with L. crispatus, are associated with a lack of genital inflammation relative to other bacterial communities. ${ }^{11,12,16,17}$ In a cross-sectional study, women with L. crispatus-dominated microbiota were less likely to be HIV positive compared with women with vaginal microbiota ei- ther dominated by L. iners or depleted of Lactobacillus spp. ${ }^{40}$ Furthermore, HIV was associated with a high bacterial load and abundance of strict and facultative anaerobes. ${ }^{40}$ While this cross-sectional analysis could be due to reverse causation (i.e., HIV could cause microbiome differences), this observation is supported by a prospective study in South African adolescent girls where $L$. crispatus-dominated cervicovaginal microbiota, but not $L$. iners, were associated with a decreased risk of acquiring HIV. ${ }^{17}$ In addition, L. iners was shown to be mildly inflammatory in in vitro cocultures with vaginal epithelial cells. ${ }^{17}$ Thus, while some Lactobacillus spp. are associated with decreased genital inflammation and HIV risk, not all Lactobacillus spp. are equally protective.

The difference in the ability of distinct Lactobacillus spp. to provide protection against HIV may be due to several factors that include their ability to produce lactic acid that is responsible for acidifying the vagina to a low $\mathrm{pH}^{25,41-43}$ Lactic acid has been shown to have antimicrobial and immune modulatory properties. ${ }^{44-47}$ Modulation of inflammatory responses by Lactobacillus spp. may also be influenced by differences in cell wall properties between strains. ${ }^{48,49}$ Another factor is the apparent lower temporal stability of L. iners-dominated microbiota compared with L. crispatusdominated microbiota. Indeed, when exposed to extrinsic and intrinsic factors, $L$. iners-dominated vaginal microbiota often transition to bacterial communities lacking Lactobacillus spp. and comprising a wide array of strict and facultative anaerobes. ${ }^{24,50}$ It is important to note that there is likely to be strain differences among Lactobacillus spp. (including L. crispatus) with levels of genital inflammation (Chetwin et al., Sci Reports, in press) as well as G. vaginalis clades and HIV risk, which cannot be resolved by Nugent scoring or current $16 \mathrm{~S}$ rRNA gene sequencing or qPCR approaches.

\section{Asymptomatic Nugent-BV or Molecular-BV Is Still Associated with Genital Inflammation}

There is considerable controversy in the field regarding asymptomatic cases that lack Lactobacillus spp. as these appear disease free but may retain elevated risk of adverse health outcomes. Women who do not report any symptoms of BV but are positive for either Nugent-BV or Molecular-BV can still have "asymptomatic BV,",35,51 a state often associated with cervicovaginal microbiota dominated by G. vaginalis (e.g., CT3; Table 1$)^{17,24}$ or are polymicrobial comprising facultative and/or obligate anaerobes while lacking Lactobacillus spp. often described as community state type IV (CST-IV), ${ }^{25} \mathrm{CT} 4,{ }^{17}$ or compositional subtype 1 $(\mathrm{C} 1)^{12,52}$ (Table 1). However, BV, including asymptomatic Molecular-BV or Nugent-BV, is often observed in African and Hispanic women, ${ }^{17,25,28,51}$ suggesting that genetic, socioeconomic, cultural, or behavioral factors might play a role alone or in combination. Furthermore, report of symptoms is subjective and varies among women since these may be "normal" if a woman has had them throughout her entire adult life. Yet her risk of HIV infection and other sexual and reproductive health outcomes may still be elevated due to asymptomatic Nugent-BV or Molecular-BV. In Gosmann et al., the majority of women who acquired HIV were asymptomatic and negative for Nugent-BV, despite having evidence of Molecular-BV. ${ }^{17}$ Thus, reliance on symptoms alone is not recommended for assessing increased risk for 
HIV. In the future, if methods to positively and effectively alter the microbiota are achieved, screening asymptomatic women in clinical practice may also be appropriate.

\section{Terminology, Definitions, and Recommendations}

We list terminologies often used in the cervicovaginal microbiome field and provide definitions as a guide for investigators to promote precision and consistency (Box 1,
Tables 1 and 2). We also propose the following recommendations for the field to consider.

1. There is a preferred consensus developing around the term "optimal" to describe cervicovaginal microbiota often associated with favorable health outcomes and characterized by a lack of symptoms, dominance of non-L. iners Lactobacillus spp., and a lack of genital inflammation. "Nonoptimal" is preferred to

Table 2. Descriptive Terms for Cervicovaginal Microbiota

\begin{tabular}{|c|c|}
\hline & Definition \\
\hline \multicolumn{2}{|l|}{ Terms for optimal microbiota } \\
\hline Optimal & $\begin{array}{l}\text { Microbiota associated with no vaginal symptoms, lack of genital inflammation } \\
\text { and favorable sexual and reproductive health outcomes, including decreased } \\
\text { risk of HIV acquisition }\end{array}$ \\
\hline Eubiosis & Microbiota that are "optimal" \\
\hline Healthy & Microbiota that are "optimal" \\
\hline Normal & $\begin{array}{l}\text { Often used to describe "optimal" microbiota; less preferred terminology since } \\
\text { "normal" is difficult to define }\end{array}$ \\
\hline Lactobacillus-dominant & $\begin{array}{l}\text { Microbiota dominated by Lactobacillus spp. usually determined by } 16 \mathrm{~S} \text { rRNA } \\
\text { gene sequencing }\end{array}$ \\
\hline Beneficial lactobacilli & $\begin{array}{l}\text { Optimal Lactobacillus spp., often used to distinguish L. crispatus (optimal) } \\
\text { from L. iners }\end{array}$ \\
\hline Protective & $\begin{array}{l}\text { Microbiota that protects against adverse health outcomes such as HIV; } \\
\text { evidence of in vivo protection is required }\end{array}$ \\
\hline Non-BV & Microbiota composed of bacteria not consistent with BV \\
\hline Microflora & $\begin{array}{l}\text { Outdated terminology that should not be used to describe microbiota; suggests } \\
\text { that microbiota are composed of plants rather than bacteria, fungi, viruses, } \\
\text { archaea, and protists }\end{array}$ \\
\hline \multicolumn{2}{|l|}{ Terms for nonoptimal microbiota } \\
\hline Nonoptimal & $\begin{array}{l}\text { Microbiota associated with vaginal symptoms, and/or genital inflammation } \\
\text { and/or adverse sexual and reproductive health outcomes, including } \\
\text { increased risk of HIV acquisition }\end{array}$ \\
\hline Dysbiosis & $\begin{array}{l}\text { Imbalance in the microbiota or impaired microbiota or "nonoptimal" } \\
\text { microbiota; avoid using this terminology for women with asymptomatic BV } \\
\text { and low levels of genital inflammation as their microbiota might represent } \\
\text { their "normal" state and may inappropriately be stigmatizing }\end{array}$ \\
\hline Low-diversity anaerobic dysbiosis & $\begin{array}{l}\text { G. vaginalis- or A. vaginae-dominated microbiota associated with adverse } \\
\text { sexual and reproductive health outcomes }\end{array}$ \\
\hline High-diversity anaerobic dysbiosis & $\begin{array}{l}\text { Polymicrobial community depleted of Lactobacillus spp. associated with } \\
\text { adverse sexual and reproductive health outcomes }\end{array}$ \\
\hline Harmful & $\begin{array}{l}\text { Less preferred terminology for microbiota associated with vaginal symptoms, } \\
\text { genital inflammation, and/or an increased risk of adverse sexual and } \\
\text { reproductive health outcomes }\end{array}$ \\
\hline Non-Lactobacillus-dominant & Non-Lactobacillus spp.-dominated bacterial community \\
\hline Polymicrobial & $\begin{array}{l}\text { Multiple bacterial species usually depleted of Lactobacillus spp. with an } \\
\text { increase in obligate and/or facultative anaerobes }\end{array}$ \\
\hline Diverse & $\begin{array}{l}\text { Used to describe microbial communities comprising multiple bacterial species } \\
\text { in the ecosystem; needs to be defined since meaning can be ambiguous with } \\
\text { respect to 16S rRNA gene sequencing }\end{array}$ \\
\hline Susceptible & $\begin{array}{l}\text { Microbiota associated with increased risk of HIV and other STIs or adverse } \\
\text { reproductive health outcomes; requires evidence to link microbiota with } \\
\text { adverse health outcomes }\end{array}$ \\
\hline MAGI & $\begin{array}{l}\text { Microbiome associated with genital inflammation; this term can also } \\
\text { encompass STIs and other microbes associated with genital inflammation, } \\
\text { including Candida spp.; requires evidence linking microbiota with genital } \\
\text { inflammation }\end{array}$ \\
\hline MAHA & $\begin{array}{l}\text { Microbiome associated with HIV acquisition; this term can also encompass } \\
\text { STIs and other microbes associated with genital inflammation, including } \\
\text { Candida spp.; requires evidence linking microbiota with increased HIV risk }\end{array}$ \\
\hline Pathobionts & $\begin{array}{l}\text { Symbiotic organism under normal circumstances that becomes pathogenic, } \\
\text { e.g., Proteobacteria, Streptococci, Staphylococci, or Enterococci spp. }\end{array}$ \\
\hline
\end{tabular}

$\mathrm{BV}$, bacterial vaginosis; MAGI, microbiota associated with genital inflammation; MAHA, microbiota associated with HIV acquisition; STIs, sexually transmitted infections. 
describe microbiota associated with adverse sexual and reproductive health outcomes, including increased HIV acquisition risk (Fig. 1).

2. Avoid use of the terms "dysbiotic" or "abnormal" microbiota, since both of these terms imply divergence from a normal state that might not exist for all women. For women with asymptomatic BV and low levels of genital inflammation, their microbial community might represent their "normal" microbial state, and these terms may inappropriately stigmatize these women.

3. We suggest that descriptive terms to describe the microbiota (i.e., in recommendations 1 and 2) be tested for acceptability with women in qualitative studies such that terminology is friendly to women who may be likely to benefit from the development of approaches to reverse the consequences of Molecular-BV.

4. We propose new terminology that specifies the method used to diagnose BV-that is, Amsel-BV (based on Amsel criteria), Nugent-BV (defined by Nugent score), and Molecular-BV (based on molecular methods)with subcategories defining the molecular technique employed, that is, Seq-BV or qPCR-BV (Box 1). Appropriate abbreviations could also be used for "nonoptimal" microbiota identified through new and emerging technologies, including metagenomics, transcriptomics, metabolomics, and metaproteomics.

5. Amsel-BV, Nugent-BV, and Molecular-BV can be further delineated into symptomatic or asymptomatic. Studies based on stratification of symptomatic BV is not recommended, given that "symptoms" can be subjective and do not fully capture the cervicovaginal microbiota associated with important health outcomes.

6. Not all "nonoptimal" microbiota are "highly diverse," that is, G. vaginalis-dominated microbiota (e.g., CT3, CST-IVB), ${ }^{11,17,24,25}$ which has also been referred to as "low-diversity anaerobic dysbiosis" as distinct from "high-diversity anaerobic dysbiosis," such as CT4 and CST-IVC. ${ }^{35}$ However, it is important to be precise when using the term "diverse" to describe microbiota. The use of the term "diverse" can be ambiguous with respect to $16 \mathrm{~S}$ rRNA gene sequencing data. It is often used to describe communities such as CT4 and CSTIVC that have "species richness," that is, many different species in a microbial ecosystem and "evenness," that is, not dominated by particular species. However, it is possible that a community dominated by L. crispatus (e.g., CT1, CST-I, C2) can have high within-community intraspecies diversity (Ravel, unpublished). In addition, "L. crispatus-dominated" microbiota could also be diverse, as a result of diversity due to very low abundance taxa representing $<1 \%$ of the community (i.e., an uneven community).

7. When describing Lactobacillus spp. as "optimal" or "beneficial," specify the Lactobacillus species. Not all Lactobacillus spp. or strains make "optimal" cervicovaginal microbiota. Current data indicate that most strains of $L$. iners are less stable, ${ }^{24}$ associated with increased genital inflammation, ${ }^{17}$ and encode factors that may be harmful to the vaginal mucosa. ${ }^{53,54}$

8. We propose terminology that describes cervicovaginal microbiota associated with genital inflammation (MAGI) and microbiota associated with HIV acquisition (MAHA). While the focus of this Perspectives article is on $\mathrm{BV}$, these terms would also encompass STIs, pathobionts, and VVC. Use of these terminologies, including "susceptible" for HIV, requires that there is evidence that the cervicovaginal microbiota increase genital inflammation (MAGI) and/or HIV risk (MAHA). These are overlapping but distinct microbiota-associated phenotypes (Fig. 1).

9. There is a need for standardization of methodology and terminology for characterizing bacterial communities by $16 \mathrm{~S}$ rRNA gene sequencing (e.g., CSTs, CTs, and Cs) defined by clustering analysis preferably compared with a reference database comprising a large number of cervicovaginal microbiota to avoid collapsing of distinct clusters due to low number of samples being analyzed. Such a database (data from 12,000 samples) has been established by Jacques Ravel, which will be made available for use (unpublished). Other areas of standardization are sample site (e.g., vaginal, cervical, and lavage), sample processing, and the use of primers directed at the same $16 \mathrm{~S}$ rRNA gene region for amplification.

10. More frequent sampling of cervicovaginal microbiota is recommended when determining the association of a cervicovaginal microbiota state with adverse health outcomes as well as more rigorous controlling of confounders that are associated with genital inflammation, including STIs and VVC.

12. Advance scientific knowledge into the mechanisms that underpin epidemiological associations observed with distinct microbial communities and HIV risk that is critical for driving the development of viable treatment and prevention modalities to promote an optimal microbiota and prevent HIV. Develop better tissue and animal models that recapitulate the FRT and can be colonized with women's cervicovaginal microbiota and infected with HIV.

\section{Conclusions}

Regardless of how it is defined, it is clear that BV is a topic of growing interest and importance for sexual and reproductive health in women. To facilitate making sense of this expanding research effort, we propose using standardized definitions that "best capture genital inflammation and/or HIV/STI risk." On this basis, molecular methods for characterizing the cervicovaginal microbiota are anticipated to replace both Nugent and Amsel as a BV gold standard. This does not imply that Nugent and Amsel no longer have a role in assessing clinical $\mathrm{BV}$. In clinical practice, Amsel will remain useful for diagnosing symptomatic BV; however, new sensitive and specific molecular diagnostic tests are becoming available, such as the FDA-approved BD MAX vaginal panel. ${ }^{33,34}$ Many properly trained sites may opt to continue using Nugent-BV due to cost or logistical reasons, since there is a plethora of data published on Nugent-BV, and it is known to capture a proportion of individuals colonized with abundance of non-Lactobacillusdominated bacterial communities with high specificity. However, to really understand the role of the nonoptimal cervicovaginal microbiota in HIV and inflammation, it will be necessary to employ a range of "omic" techniques, including metagenomics (next-generation DNA sequencing of whole 
bacteria, not only the 16S rRNA gene), transcriptomics, proteomics, and metabolomics, in conjunction with immunological measurements. Use of these techniques will be necessary to advance our knowledge of $\mathrm{BV}$ and conditions that promote $\mathrm{BV}$ so that better treatments can be developed and to stop the cycle of frequent recurrence that is commonplace with current treatments.

\section{Acknowledgments}

A.D.B. and L.R.M. are funded by the Canadian Institutes of Health Research (CIHR) grant TMI-138658. L.R.M. is supported by a CIHR New Investigator award and A.D.B. by the CIHR New Investigator in HIV award NIH-15404. R.K. is funded by CIHR grant PJT-156123. C.K. is funded by an Ontario HIV Treatment Network Applied HIV Chair award and operating grants and an HIV Team grant from CIHR. J.R. was supported by the National Institute for Allergy and Infectious Diseases of the National Institutes of Health under award number U19AI084044. G.T. is funded by the National Health and Medical Research Council of Australia Senior Research Fellowship GNT1117748. G.T. gratefully acknowledges the contribution of the Victorian Operational Infrastructure Support Program received by the Burnet Institute.

\section{Author Disclosure Statement}

D.N.F. declares intellectual property around the molecular diagnosis of $\mathrm{BV}$ and receives royalty from BD. J.M.M. is a consultant for Biofire, receives research supplies from Merck and Toltec Pharmaceuticals, and serves on the DSMB for Gilead. G.T. is a coinventor on patent application AU201501042 and United States Patent No. US 9,801,839 B2 claiming the anti-inflammatory effects of lactic acid. The remaining authors have no competing financial interests to declare.

\section{References}

1. Libertucci J, Young VB: The role of the microbiota in infectious diseases. Nat Microbiol 2019;4:35-45.

2. Burgener A, McGowan I, Klatt NR: HIV and mucosal barrier interactions: Consequences for transmission and pathogenesis. Curr Opin Immunol 2015;36:22-30.

3. Ma B, Forney LJ, Ravel J: Vaginal microbiome: Rethinking health and disease. Annu Rev Microbiol 2012;66:371-389.

4. Marrazzo JM, Martin DH, Watts DH, et al.: Bacterial vaginosis: Identifying research gaps proceedings of a workshop sponsored by DHHS/NIH/NIAID. Sex Transm Dis 2010;37:732-744.

5. Martin DH, Marrazzo JM: The vaginal microbiome: Current understanding and future directions. J Infect Dis 2016; 214 Suppl 1:S36-S41.

6. Aldunate M, Srbinovski D, Hearps AC, et al.: Antimicrobial and immune modulatory effects of lactic acid and short chain fatty acids produced by vaginal microbiota associated with eubiosis and bacterial vaginosis. Front Physiol 2015;6:164.

7. Cohen CR, Lingappa JR, Baeten JM, et al: Bacterial vaginosis associated with increased risk of female-to-male HIV-1 transmission: A prospective cohort analysis among African couples. PLoS Med 2012;9:e1001251.

8. Kenyon C, Colebunders R, Crucitti T: The global epidemiology of bacterial vaginosis: A systematic review. Am J Obstet Gynecol 2013;209:505-523.
9. Amsel R, Totten PA, Spiegel CA, Chen KC, Eschenbach D, Holmes KK: Nonspecific vaginitis. Diagnostic criteria and microbial and epidemiologic associations. Am J Med 1983; 74:14-22.

10. Mitchell C, Marrazzo J: Bacterial vaginosis and the cervicovaginal immune response. Am J Reprod Immunol 2014; 71:555-563.

11. Anahtar $\mathrm{MN}$, Byrne $\mathrm{EH}$, Doherty $\mathrm{KE}$, et al.: Cervicovaginal bacteria are a major modulator of host inflammatory responses in the female genital tract. Immunity 2015;42:965-976.

12. Lennard K, Dabee S, Barnabas SL, et al:: Microbial composition predicts genital tract inflammation and persistent bacterial vaginosis in South African adolescent females. Infect Immun 2018;86:e00410-17.

13. Gautam R, Borgdorff H, Jespers V, et al.: Correlates of the molecular vaginal microbiota composition of African women. BMC Infect Dis 2015;15:86.

14. Masson L, Mlisana K, Little F, et al.: Defining genital tract cytokine signatures of sexually transmitted infections and bacterial vaginosis in women at high risk of HIV infection: A cross-sectional study. Sex Transm Infect 2014;90:580-587.

15. Deese J, Masson L, Miller W, et al..: Injectable progestinonly contraception is associated with increased levels of pro-inflammatory cytokines in the female genital tract. Am J Reprod Immunol 2015;74:357-367.

16. Kyongo JK, Crucitti $\mathrm{T}$, Menten J, et al.: Cross-sectional analysis of selected genital tract immunological markers and molecular vaginal microbiota in sub-Saharan African women, with relevance to HIV risk and prevention. Clin Vaccine Immunol 2015;22:526-538.

17. Gosmann C, Anahtar MN, Handley SA, et al.: Lactobacillus-deficient cervicovaginal bacterial communities are associated with increased HIV acquisition in young South African women. Immunity 2017;46:29-37.

18. Atashili J, Poole C, Ndumbe PM, Adimora AA, Smith JS: Bacterial vaginosis and HIV acquisition: A meta-analysis of published studies. AIDS 2008;22:1493-1501.

19. Low N, Chersich MF, Schmidlin K, et al.: Intravaginal practices, bacterial vaginosis, and HIV infection in women: Individual participant data meta-analysis. PLoS Med 2011; 8:e1000416.

20. Nugent RP, Krohn MA, Hillier SL: Reliability of diagnosing bacterial vaginosis is improved by a standardized method of gram stain interpretation. J Clin Microbiol 1991; 29:297-301.

21. Srinivasan S, Morgan MT, Liu C, et al:: More than meets the eye: Associations of vaginal bacteria with gram stain morphotypes using molecular phylogenetic analysis. PLoS One 2013;8:e78633.

22. Wessels JM, Lajoie J, Vitali D, et al.: Association of high-risk sexual behaviour with diversity of the vaginal microbiota and abundance of Lactobacillus. PLoS One 2017;12:e0187612.

23. Kroon SJ, Ravel J, Huston WM: Cervicovaginal microbiota, women's health, and reproductive outcomes. Fertil Steril 2018;110:327-336.

24. Gajer P, Brotman RM, Bai G, et al.: Temporal dynamics of the human vaginal microbiota. Sci Transl Med 2012;4:132ra52.

25. Ravel J, Gajer P, Abdo Z, et al.: Vaginal microbiome of reproductive-age women. Proc Natl Acad Sci U S A 2011; 108 Suppl 1:4680-4687.

26. Fredricks DN, Fiedler TL, Marrazzo JM: Molecular identification of bacteria associated with bacterial vaginosis. N Engl J Med 2005;353:1899-1911. 
27. Fettweis JM, Serrano MG, Sheth NU, et al.: Species-level classification of the vaginal microbiome. BMC Genomics 2012;13 Suppl 8:S17.

28. Srinivasan S, Hoffman NG, Morgan MT, et al.: Bacterial communities in women with bacterial vaginosis: High resolution phylogenetic analyses reveal relationships of microbiota to clinical criteria. PLoS One 2012;7:e37818.

29. McClelland RS, Lingappa JR, Srinivasan S, et al:: Evaluation of the association between the concentrations of key vaginal bacteria and the increased risk of HIV acquisition in African women from five cohorts: A nested case-control study. Lancet Infect Dis 2018;18:554-564.

30. Zevin AS, Xie IY, Birse K, et al.: Microbiome composition and function drives wound-healing impairment in the female genital tract. PLoS Pathog 2016;12:e1005889.

31. Klatt NR, Cheu R, Birse K, et al.: Vaginal bacteria modify HIV tenofovir microbicide efficacy in African women. Science 2017;356:938-945.

32. Borgdorff $\mathrm{H}$, Gautam R, Armstrong SD, et al.: Cervicovaginal microbiome dysbiosis is associated with proteome changes related to alterations of the cervicovaginal mucosal barrier. Mucosal Immunol 2016;9:621-633.

33. Gaydos CA, Beqaj S, Schwebke JR, et al:: Clinical validation of a test for the diagnosis of vaginitis. Obstet Gynecol 2017;130:181-189.

34. Schwebke JR, Gaydos CA, Nyirjesy P, Paradis S, Kodsi S, Cooper CK: Diagnostic performance of a molecular test versus clinician assessment of vaginitis. J Clin Microbiol 2018;56:e00252-18.

35. van de Wijgert JHHM: The vaginal microbiome and sexually transmitted infections are interlinked: Consequences for treatment and prevention. PLoS Med 2017; 14:e1002478.

36. Donders GG, Ruban K, Bellen G: Selecting anti-microbial treatment of aerobic vaginitis. Curr Infect Dis Rep 2015;17:477.

37. van de Wijgert $\mathrm{JH}$, Borgdorff $\mathrm{H}$, Verhelst $\mathrm{R}$, et al.: The vaginal microbiota: What have we learned after a decade of molecular characterization? PLoS One 2014;9:e105998.

38. Arnold KB, Burgener A, Birse K, et al.: Increased levels of inflammatory cytokines in the female reproductive tract are associated with altered expression of proteases, mucosal barrier proteins, and an influx of HIV-susceptible target cells. Mucosal Immunol 2016;9:194-205.

39. Shannon B, Gajer P, Yi TJ, et al.: Distinct effects of the cervicovaginal microbiota and herpes simplex type 2 infection on female genital tract immunology. J Infect Dis 2017;215:1366-1375.

40. Borgdorff $\mathrm{H}$, Tsivtsivadze $\mathrm{E}$, Verhelst $\mathrm{R}$, et al.: Lactobacillus-dominated cervicovaginal microbiota associated with reduced HIV/STI prevalence and genital HIV viral load in African women. ISME J 2014;8:1781-1793.

41. O'Hanlon DE, Moench TR, Cone RA: Vaginal $\mathrm{pH}$ and microbicidal lactic acid when lactobacilli dominate the microbiota. PLoS One 2013;8:e80074.

42. Witkin SS, Mendes-Soares H, Linhares IM, Jayaram A, Ledger WJ, Forney LJ: Influence of vaginal bacteria and Dand L-lactic acid isomers on vaginal extracellular matrix metalloproteinase inducer: Implications for protection against upper genital tract infections. MBio 2013;4:e00460-13.

43. Tyssen D, Wang YY, Hayward JA, et al.: Anti-HIV-1 activity of lactic acid in human cervicovaginal fluid. $\mathrm{mSphere}$ 2018;3:e0055-18.
44. Tachedjian G, Aldunate M, Bradshaw CS, Cone RA: The role of lactic acid production by probiotic Lactobacillus species in vaginal health. Res Microbiol 2017; 168:782-792.

45. Aldunate M, Tyssen D, Johnson A, et al.: Vaginal concentrations of lactic acid potently inactivate HIV. J Antimicrob Chemother 2013;68:2015-2025.

46. Tachedjian G, O'Hanlon DE, Ravel J: The implausible "in vivo" role of hydrogen peroxide as an antimicrobial factor produced by vaginal microbiota. Microbiome 2018;6:29.

47. Hearps AC, Tyssen D, Srbinovski D, et al..: Vaginal lactic acid elicits an anti-inflammatory response from human cervicovaginal epithelial cells and inhibits production of pro-inflammatory mediators associated with HIV acquisition. Mucosal Immunol 2017;10:1480-1490.

48. Chapot-Chartier MP, Kulakauskas S: Cell wall structure and function in lactic acid bacteria. Microb Cell Fact 2014; 13 Suppl 1:S9.

49. Macho Fernandez E, Valenti V, Rockel C, et al:: Antiinflammatory capacity of selected lactobacilli in experimental colitis is driven by NOD2-mediated recognition of a specific peptidoglycan-derived muropeptide. Gut 2011;60: 1050-1059.

50. Ravel J, Brotman RM, Gajer P, et al.: Daily temporal dynamics of vaginal microbiota before, during and after episodes of bacterial vaginosis. Microbiome 2013;1:29.

51. Masson L, Barnabas S, Deese J, et al: Inflammatory cytokine biomarkers of asymptomatic sexually transmitted infections and vaginal dysbiosis: A multicentre validation study. Sex Transm Infect 2019;95:5-12.

52. Balle C, Lennard K, Dabee S, et al:: Endocervical and vaginal microbiota in South African adolescents with asymptomatic Chlamydia trachomatis infection. Sci Rep 2018;8:11109.

53. Macklaim JM, Fernandes AD, Di Bella JM, Hammond JA, Reid G, Gloor GB: Comparative meta-RNA-seq of the vaginal microbiota and differential expression by Lactobacillus iners in health and dysbiosis. Microbiome 2013;1:12.

54. Petrova MI, Reid G, Vaneechoutte M, Lebeer S: Lactobacillus iners: Friend or Foe? Trends Microbiol 2017;25: 182-191.

Address correspondence to: Gilda Tachedjian, BSc (Hons), PhD Disease Elimination Program Life Sciences Discipline Burnet Institute 85 Commercial Road Melbourne 3004 Australia

E-mail: gilda.tachedjian@burnet.edu.au

Lyle R. McKinnon, BSc, PhD

Department of Medical Microbiology and Infectious Diseases University of Manitoba

Room 504, 745 Bannatyne Avenue Winnipeg MB R3E OJ9 Canada

E-mail: lyle.mckinnon@umanitoba.ca 